\title{
MPC polymer regulates fibrous tissue formation by modulating cell adhesion to the biomaterial surface
}

\author{
Ye ZHANG ${ }^{1}$, Hiroyasu KANETAKA ${ }^{1,2}$, Yuya SANO ${ }^{2}$, Mitsuhiro KANO ${ }^{3}$, Tada-aki KUDO ${ }^{4}$ and Yoshinaka SHIMIZU ${ }^{3}$ \\ ${ }^{1}$ Department of Physical Medicine and Rehabilitation, Graduate School of Biomedical Engineering, Tohoku University, 4-1 Seiryo-machi, Aoba-ku, \\ Sendai 980-8575, Japan \\ ${ }^{2}$ Division of Advanced Prosthetic Dentistry, Tohoku University Graduate School of Dentistry, Tohoku University, 4-1 Seiryo-machi, Aoba-ku, Sendai \\ 980-8575, Japan \\ ${ }^{3}$ Division of Oral and Craniofacial Anatomy, Tohoku University Graduate School of Dentistry, Tohoku University, 4-1 Seiryo-machi, Aoba-ku, Sendai \\ 980-8575, Japan \\ ${ }^{4}$ Division of Oral Physiology, Graduate School of Dentistry, Tohoku University, 4-1 Seiryo-machi, Aoba-ku, Sendai 980-8575, Japan \\ Corresponding author, Ye ZHANG; E-mail: zhangye@m.tains.tohoku.ac.jp
}

\begin{abstract}
The aim of this study was to analyze the effects of 2-methacryloyloxyethyl phosphorylcholine (MPC) polymer on fibrous tissue formation and cell adhesion plaque (CAP)-forming reactions. Silastic elastomer (SE) plates coated (experimental group) and uncoated (control group) with MPC polymer were prepared for in vivo and in vitro experiments. For the in vivo animal experiments, SE plates were implanted subcutaneously in the rat dorsal region. At 4, 8, and 12 weeks, thicknesses of the fibrous tissue capsules in the experimental group were lower than in the control group. Likewise, the amount of collagen in the experimental group was lower than that of the control group. For the in vitro cell culture experiments, KMST-6 fibroblast cells in the experimental group demonstrated enhanced cell migration, accompanied with a weaker expression of vinculin and a larger amount of filopodia. Furthermore, weaker expressions of paxillin, talin, and ROCK1, but stronger expression of cofilin, were observed in the experimental group. Taken together, these results suggested that MPC polymer regulated fibrous tissue formation by modulating cell adhesion through changes in local CAPs and downstream signaling.
\end{abstract}

Keywords: 2-methacryloyloxyethyl phosphorylcholine polymer, Fibrous tissue, Cell adhesion plaque

\section{INTRODUCTION}

For the treatment of diseases such as cancer, trauma and myocardial infarction, numerous biomaterials and artificial devices have been used effectively as treatment methods ${ }^{11}$. Despite the laudable treatment success achieved with these biomaterials, the downside is that biomaterials implanted into the body frequently induce inflammation and immune responses, often leading to fibrous scar formation ${ }^{2)}$. Although these reactions are a result of normal histological repair responses to foreign bodies, active scar formation may result in unfavorable outcomes, such as breast collapse after prosthetic implantation ${ }^{3)}$ and stiff joints after artificial joint replacement ${ }^{4}$. Against this backdrop of undesirable outcomes, an important consideration prior to and pertaining to the use of biomaterials and artificial devices is the inhibition of fibrous tissue formation.

When a biomaterial is implanted into a body, it induces a complex sequence of biological reactions in the local tissue, including cell contact, recognition, and signal transduction. The first event in vivo is cell migration to the biomaterial, and hence cell contact with the biomaterial ${ }^{5}$. In light of this cellular phenomenon, the material surface is an important factor in biocompatibility estimation since it affects cell reactions and signal transduction. To the end of fabricating blood-compatible polymer surfaces, a novel coating material based on natural phospholipid cell membranes has been proposed ${ }^{6}$.

2-methacryloyloxyethyl phosphorylcholine (MPC), which has a phospholipid polar group that mimics a biomembrane, was synthesized as a novel coating material by Ishihara et $a l .{ }^{7}$. Numerous studies have shown that MPC is suitable for use as a biomaterial due to benefits such as suppressing protein adsorption, platelet adhesion, and denaturation of cells and proteins ${ }^{8}$. However, MPC has several drawbacks, amongst which are poor mechanical properties caused by the introduction of water-soluble moieties, and in the case of coatings, weak bonding to the substrate leading to delamination ${ }^{6}$. To improve its mechanical properties and reduce the proneness to delamination, MPC may be copolymerized with other methacrylate monomers, such as $n$-butyl methacrylate (BMA), $n$ hexyl methacrylate, and $n$-dodecyl methacrylate $(\mathrm{DMA})^{9)}$, through atom transfer radical polymerization (ATRP) or conventional free radical polymerization initiated by different methods ${ }^{10)}$.

Having circumvented the mechanical weaknesses through copolymerization, MPC polymers have since become attractive candidates for artificial organs and have shown potential in a wide range of medical device applications. For example, Kyomoto et al. reported its use in artificial hip joints, enhancing lubrication to reduce wear and prosthesis loosening ${ }^{11)}$. Huang et al. used it in soft contact lenses to prevent protein adsorption ${ }^{12)}$. The list goes on with MPC polymer being applied to myriad medical devices including 
biosensors ${ }^{13)}$, drug carriers, vascular stents, and urological devices ${ }^{6}$.

Owing to its ability to mimic the biomembrane, MPC polymer might possess the capacity to regulate unwanted fibrous tissue formation. To date, the evaluation of MPC polymer as a potential coating material for biomaterials has been limited to the analyses of its chemical properties and blood compatibility $^{14)}$. This meant that the biocompatibility of MPC polymer, particularly its ability to inhibit fibrous tissue formation and cell adhesion, has not been conclusively demonstrated through in vivo and in vitro experiments. Nonetheless, it has been shown that MPC polymer influenced cell reactions. Konno et al. reported that cell mobility on MPC polymer surface was affected ${ }^{15)}$ by its less adhesive surface, leading to accelerated cell aggregation and differentiation. Incidentally, cell adhesion plaque (CAP) plays an essential role in fibrous tissue formation and cell mobility ${ }^{16)}$. For this reason, we hypothesized that the MPC biomembrane could regulate the downstream signals of CAP to suppress protein adsorption and alter cell viability, thereby inhibiting fibrous tissue formation.

Silastic elastomers (SE) are very commonly used in maxillofacial prostheses due to their superior physical properties ${ }^{17)}$. However, they are not without shortcomings ${ }^{18)}$-amongst which are the tendency to induce fibrous tissue formation, absorb facial oils which can adversely impact prosthesis longevity ${ }^{19)}$, and to provide a potential site for bacterial colonization. Nonetheless, with improved biocompatibility bestowed upon it by means of MPC polymer coating, silastic elastomers (SE) are well poised to be used in a wider range of medical applications. For this reason, silastic elastomer (SE) was selected in this study as the substrate material to be coated with MPC polymer. Our two-pronged experimental strategy comprised a subcutaneous implant in vivo model and in vitro cell culture to observe capsule scar formation and the regulation of downstream CAP signals, with a view to leveraging on these findings for new medical applications.

\section{MATERIALS AND METHODS}

Preparation of silastic elastomer coated with MPC polymer

This study was carried out in compliance with the guidelines of the International Organization of Standardization (ISO) 10996-3 (Biological evaluation of medical devices). MPC polymer (MPC-co-BMA) was prepared as described by Ishihara et al. (Ai Bio-Chips, Tokyo, Japan) ${ }^{20)}$ (Fig. 1). Surface coating was carried out according to the method reported by Sibarani et $a l .^{21)}$.

Plates used for MPC polymer coating were fabricated from Silastic ${ }^{\circledR}$ MDX4-4210 BioMedical Grade Elastomer (Dow Corning Chemical Industries, Osaka, Japan). Curing agent and base elastomer (1:10) were

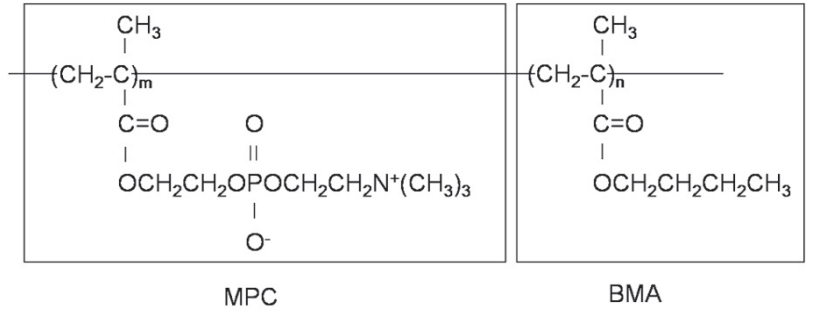

Fig. 1 Chemical formula of poly(MPC-co-BMA) (PMB, m: 0.3, n: 0.7).

thoroughly mixed and exposed to a vacuum of 710 $\mathrm{mmHg}$ for 30 minutes to remove trapped air. Then, in accordance with ISO-10996-3 recommendation ${ }^{20)}$, silastic elastomer (SE) was placed into a master mold $(2 \times 5 \times 20 \mathrm{~mm})$ and cured for 5 hours at $65^{\circ} \mathrm{C}$. SE plates were cleaned by sonication in acetone (Wako Pure Chemical Industries Osaka, Japan) and alcohol. For MPC polymer coating, each SE plate was dipped in an EtOH solution containing 0.3 wt\% MPC polymer. Solvent was slowly evaporated under an $\mathrm{EtOH}$ vapor atmosphere at room temperature for 24 hours, and SE plates were dried in vacuo overnight.

Surface analysis of SE plates coated with MPC polymer was done using X-ray photoelectron spectroscopy (XPS; AXIS His-165 Analytical, Shimadzu Corp., Kyoto, Japan). The take-off angle of the photoelectrons was $90^{\circ}$. The chemical composition of the SE plates was determined using an energy dispersive X-ray analyzer (EDX; JED-2300, JEOL, Japan) operated at an accelerating voltage of $15 \mathrm{kV}$ and with a filament current of $60 \mu \mathrm{A}$.

\section{Subcutaneous implantation in rats}

All animal experiments were performed in accordance with the Guide for the Care and Use of Laboratory Animals (Tohoku University), $3^{\text {rd }}$ edition. Twenty-four 10-week-old male SD rats were used in all the experiments. Rats were anesthetized by an intraperitoneal injection of sodium pentobarbital (30 $\mathrm{mg} / \mathrm{kg}$ ) (Wako Pure Chemical Industries, Osaka, Japan), and target regions were cleaned with $70 \%$ ethanol. Sagittal incisions of $2 \mathrm{~cm}$ were made $0.5 \mathrm{~mm}$ to the right and left of the dorsal centreline, and then subcutaneous pockets were created by blunt dissection in the thoracic region. SE plates coated and uncoated with MPC polymer $(2 \times 5 \times 20 \mathrm{~mm})$ were implanted subcutaneously in the left and right sides respectively. Skin wounds were sutured tightly using Polysorb suture No. 3 (Auto Suture, Norwalk, CT, USA).

\section{Histological evaluation of fibrous tissue formation}

Rats were anesthetized and sacrificed for histological evaluation at $2,4,8$, and 12 weeks after surgery (six rats at each time point). SE plates with surrounding tissues were resected. The SE plates were examined using EDX, while the surrounding tissues were fixed 
with $10 \%$ neutral buffered formaldehyde, followed by embedding in paraffin. Sliced sections $(3 \mu \mathrm{m})$ were routinely stained with hematoxylin and eosin (HE) to evaluate tissue reactions such as inflammation and scar formation around the plates. Thickness of fibrous tissue capsule was measured with a microscope (BX51, Olympus, Tokyo, Japan), as reported previously ${ }^{22)}$.

\section{Collagen staining and measurement}

K61 Kit (Wako, Osaka, Japan) was used to stain the collagen in samples of surrounding tissues ${ }^{21}$. Histological sections $(6 \mu \mathrm{m})$ were deparaffinized and stained with a saturated solution of picric acid in distilled water containing $0.01 \%$ Fast green FCF. Sections were kept out of the light and incubated at room temperature for 15 minutes. After which, they were stained with a saturated solution of picric acid in distilled water containing 0.04\% Fast green and 0.1\% Sirius red F3B. After incubation in the dark at room temperature for 30 minutes, the stained histological sections were observed under a polarizing microscope (DM500, Leica, Wetzlar, Germany).

To measure the collagen content, $0.1 \% \mathrm{NaOH}$ in absolute methanol (1:1, v:v, $200 \mu \mathrm{L})$ was added to the glass slide. Added $\mathrm{NaOH}$ was retrieved and transferred to a test tube, and the eluted color was read in a spectrophotometer at 530 and $605 \mathrm{~nm}$ (GloMax-Multi ${ }^{+}$ Detection System, Promega, Madison, Wisconsin, USA). The amounts of collagen and non-collagenous protein were calculated using the following formula:

\section{Collagen $(\mathrm{mg})=(O D(530)-0.254 \times O D(605)) / 40.8$}

Non-collagenous protein $(\mathrm{mg})=O D(605) / 2.04$

\section{Cell cultures on plate surfaces}

SE plates coated and uncoated with MPC polymer were placed in 6-well tissue culture plates (Thermo Fisher Scientific, Rochester, NY, USA). Human fibroblast cells (KMST-6, obtained from the Cell Bank of the Old and Aging Medical Institute, Tohoku University, Sendai, Japan) were added to each well at a concentration of $2 \times 10^{5}$ cells/well in a cell culture medium (Dulbecco Eagle's MEM; Nissui Pharmaceutical, Tokyo, Japan) containing $10 \%$ fetal bovine serum (FBS; GIBCO BRL, Invitrogen Corp., Grand island, NY, USA). KMST-6 cells were maintained in a culture medium ( $\alpha$-MEM, GIBCO BRL) containing 10\% fetal bovine serum at $37^{\circ} \mathrm{C}$ in a humidified atmosphere of air containing $5 \%$ $\mathrm{CO}_{2}$. Cells were observed daily by phase-contrast microscopy (DM500, Leica, Wetzlar).

\section{MTT assay}

Cell viability was evaluated using MTT assay ${ }^{23)}$. Briefly, coated and uncoated samples were set at the flat-bottom 96-well plates, and KMST-6 cells were seeded on it $\left(10^{4} /\right.$ well $)$ with $100 \mu \mathrm{L}$ of medium. After 24-hour incubation for attachment, the medium was replaced with a fresh serum-free medium. Then, each day from day 1 to day 6 , six wells were added with 10 $\mu \mathrm{L}$ of MTT $(5 \mathrm{mg} / \mathrm{mL}$ ) (Wako Pure Chemical Industries,
Osaka, Japan) and incubated for 4 hours. $100 \mu \mathrm{L}$ of DMSO was also added to each well and mixed. Absorbance at $492 \mathrm{~nm}$ was measured using the spectrophotometer (GloMax-Multi ${ }^{+}$Detection System, Promega, Madison, Wisconsin, USA).

Adhesion plaque staining by immunofluorescence histochemistry

KMST- 6 cells were collected at day 3 after culturing on SE plates coated and uncoated with MPC polymer. SE plates were washed in phosphate buffered saline (PBS) and fixed with $4 \%$ paraformaldehyde. SE plates were then treated with $0.2 \%$ Triton X-100. After washing with Tris-buffer (TBS, $\mathrm{pH}$ 7.6), SE plates were incubated in $100 \mu \mathrm{L}$ of blocking buffer. Anti-vinculin antibody (Sigma-Aldrich, St. Louis, MO, USA) was used at a 1:50 dilution. Plates were incubated with primary antibody for 60 minutes at room temperature, followed by incubation with rhodamine-conjugated secondary antibody (Chemicon ${ }^{\circledR}$ International, Inc., Temecula, CA, USA). After sufficient rinsing with TBS, SE plates were incubated with Alexa Fluor ${ }^{\circledR} 488$ conjugated to phalloidin (5 IU; Cambrex, Walkersville, MD, USA) for 20 minutes at room temperature. After washing, SE plates were mounted on PLA-coated glass plates with a mounting medium. Cell adhesion and the cytoskeleton were observed with a confocal laser scanning microscope (LSM 510 META, Zeiss, Jena, Germany).

mRNA expression levels of fibronectin-1 and focal adhesion kinase (FAK)

KMST-6 cells cultured on SE plates coated and uncoated with MPC polymer were harvested using lysis buffer. Total RNA was isolated using the RNeasy Mini Kit (Qiagen, Hilden, Germany). RNA concentrations were spectrophotometrically measured at $260 \mathrm{~nm}$, and RNA purity was confirmed from the ratio of absorbance at $260 \mathrm{~nm}$ to that at $280 \mathrm{~nm}$. First-strand cDNA synthesis was performed using $1 \mu \mathrm{g}$ of total RNA. RNA samples were reverse transcribed to cDNA using the SuperScript First-Strand Synthesis System (GIBCO BRL, Invitrogen Corp., NY, USA).

Real-time PCR was performed on a TP800BK Thermal Cycler Dice ${ }^{\mathrm{TM}}$ Real Time System (Takara Biochemicals, Kyoto, Japan) using a SYBR ${ }^{\circledR}$ Premix Ex Taq $^{\text {TM }}$ kit (Takara Biochemicals, Kyoto, Japan). Thermal cycling conditions were as follows: initial hold for 2 minutes at $50^{\circ} \mathrm{C}$ and 10 minutes at $95^{\circ} \mathrm{C}$, followed by 40 cycles at $95^{\circ} \mathrm{C}$ for 15 seconds and $60^{\circ} \mathrm{C}$ for 60 seconds. Primer pairs used for fibronectin-1 and FAK, as well as for the reference gene (housekeeping gene) $\beta$-actin, were designed using DINAsis software (Toshiba, Tokyo, Japan) and were produced by Nippon Gene (Sendai, Japan). The primers were fibronectin-1 (NM_212482), 5'-GAGCCATGTGTCTTACCATT-3' (forward), 5'-AGTATTTCTGGTCCTGCTCA-3' (reverse); FAK (L13616), 5'ACTTGGACGATGTATTGGAG-3' (forward), 5'-GCTGTCCGAAGTACAGTTTT-3' (reverse); and $\beta$-actin (NM_001101.3), 5'- 
CTAAGTCATAGTCCGCCTAGAAGCA-3' (forward), 5'TGGCACCCAGCACAATGAA-3' (reverse). Relativity quantities normalized against $\beta$-actin were calculated, and all reactions were performed in triplicate. Quantification of mRNA expression levels was achieved using a standard curve, as reported previously ${ }^{24)}$.

Protein expression levels of ROCK1, cofilin, paxillin, and talin

Protein expression was assessed by Western blotting analysis. Cells cultured on SE plates coated and uncoated with MPC polymer were lysed in RIPA buffer (1×TBS, 1\% Nonidet P-40, 0.5\% sodium deoxycholate, $0.1 \%$ SDS, $\quad 0.004 \%$ sodium azide, $1 \%$ sodium orthovanadate, $1 \%$ phenylmethylsufonyl fluoride (PMSF), and 1\% protease inhibitor cocktail). Lysates were centrifuged at $13,000 \mathrm{~g}$ for 5 minutes at $4^{\circ} \mathrm{C}$ to collect the supernatant. Protein concentration was measured using Bradford protein assay (Bio-Rad Laboratories, Hercules, CA, USA). Protein samples were separated by $12 \%$ sodium dodecyl sulfatepolyacrylamide gel electrophoresis (SDS-PAGE) with the Mini-PROTEAN Tetra cell (Bio-Rad Laboratories, CA, USA).

Proteins were immobilized on a polyvinylidene fluoride (PVDF) transfer membrane by electro-transfer using a Mini Trans-Blot Cell (Bio-Rad Laboratories, CA, USA). Immobilized proteins were blocked overnight with $3 \%$ milk solution in $1 \times$ phosphate buffered saline (PBS) containing $0.1 \%$ Tween 20 (PBST), and then incubated for 30 minutes with each primary antibody (anti-ROCK1, 1:1000 dilution; anticofilin, 1:1000 dilution; anti-paxillin, 1:500 dilution; and anti-talin, 1:250 dilution). Membranes were then washed with PBST and incubated with HRP-conjugated donkey anti-rabbit IgG (1:2000 in PBST with 3\% milk;
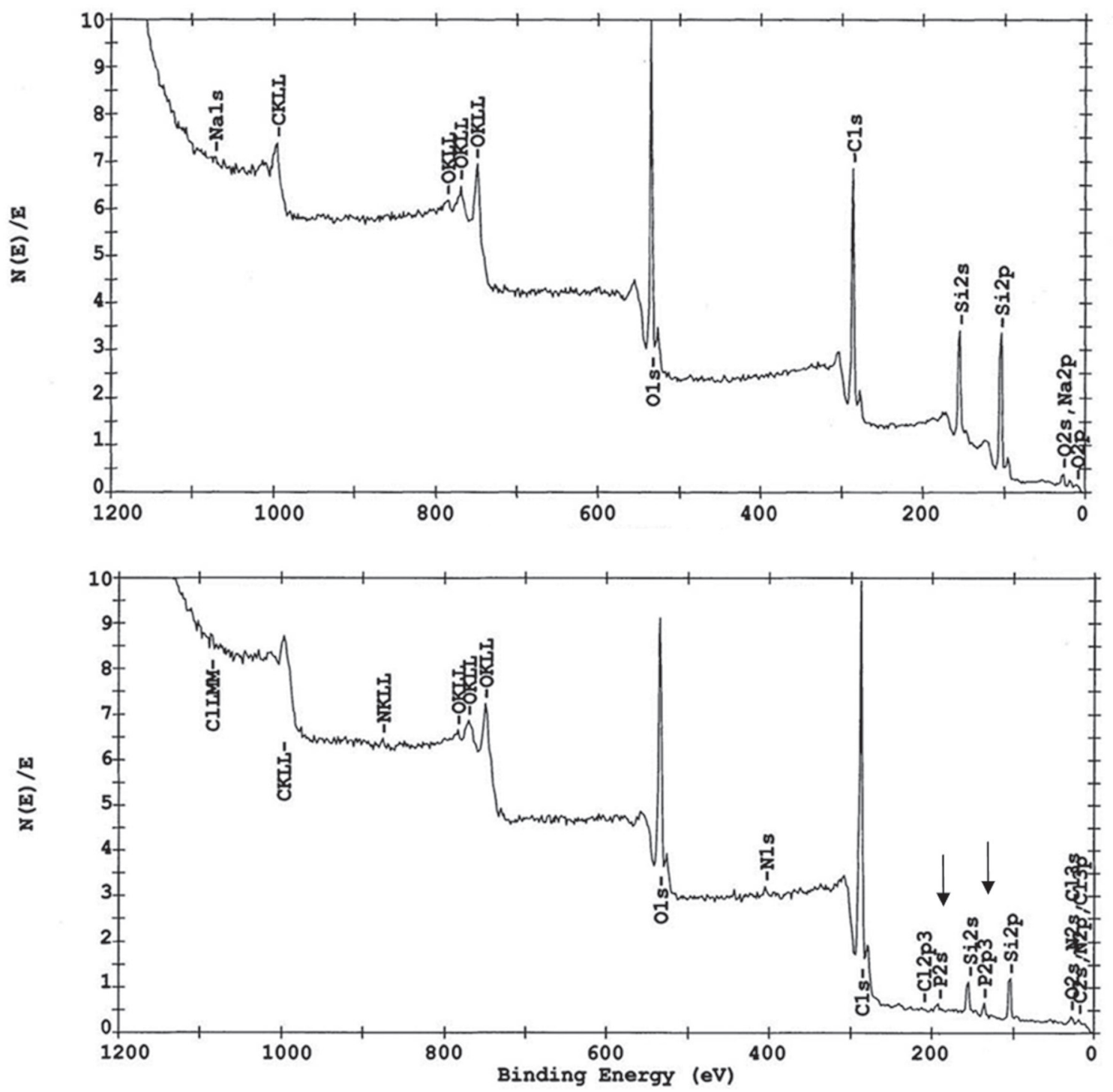

Fig. 2 XPS imaging of SE plate surfaces in control (upper) and experimental (lower) groups. $\uparrow:$ Peaks for $\mathrm{P}_{2} \mathrm{~S}$ and $\mathrm{P}_{2} \mathrm{p} 3$. 
Dako, Carpinteria, CA, USA) for 1 hour at room temperature. After washing with PBST, antibody reactivity was visualized using ECL (GE Healthcare, Buckinghamshire, UK) by exposure to X-ray film (Fujifilm Corp., Tokyo, Japan).

\section{Statistical analysis}

All data were presented as mean \pm standard deviation. Three independent cultures were used for each biochemical analysis. Statistical differences were analyzed by one-way ANOVA and Tukey's test using JSTAT 12.5 software. Differences at $p<0.05$ were considered to be statistically significant.

\section{RESULTS}

\section{XPS and EDX}

As MPC was used to simulate the surface structure of a natural biomembrane, phosphate components were investigated. On the surface of the SE plate coated with MPC polymer, peaks for $\mathrm{P}_{2} \mathrm{~S}$ and $\mathrm{P}_{2} \mathrm{p} 3$ were observed in its XPS spectrum (Fig. 2). Similarly, the corresponding EDX spectrum also revealed the peak for phosphate (Fig. 3, upper right). Taken together, these results indicated that the $\mathrm{SE}$ plate was successfully coated with MPC polymer.

After 12 weeks of implantation, EDX analysis revealed that the MPC polymer coating remained on the SE plates removed from the animals (Fig. 3, lower), indicating that the MPC polymer coating remained stable and intact throughout the entire implantation period.

\section{Histology}

At 2 weeks after implantation, SE plates in the control and experimental groups were surrounded by fibrous connective tissue with slight inflammatory cell infiltration (Fig. 4). Moreover, at 2 weeks, fibrous tissue was thin and thickness did not differ between the experimental and control groups $(n=6, p>0.05)$.

In the control group, the thickness of fibrous connective tissue increased steadily at 4 and 8 weeks (Fig. 5). Besides, capillaries and some inflammatory cells were seen around the fibrous connective tissue. At 12 weeks, dense fibrous tissue was observed around the SE plate.
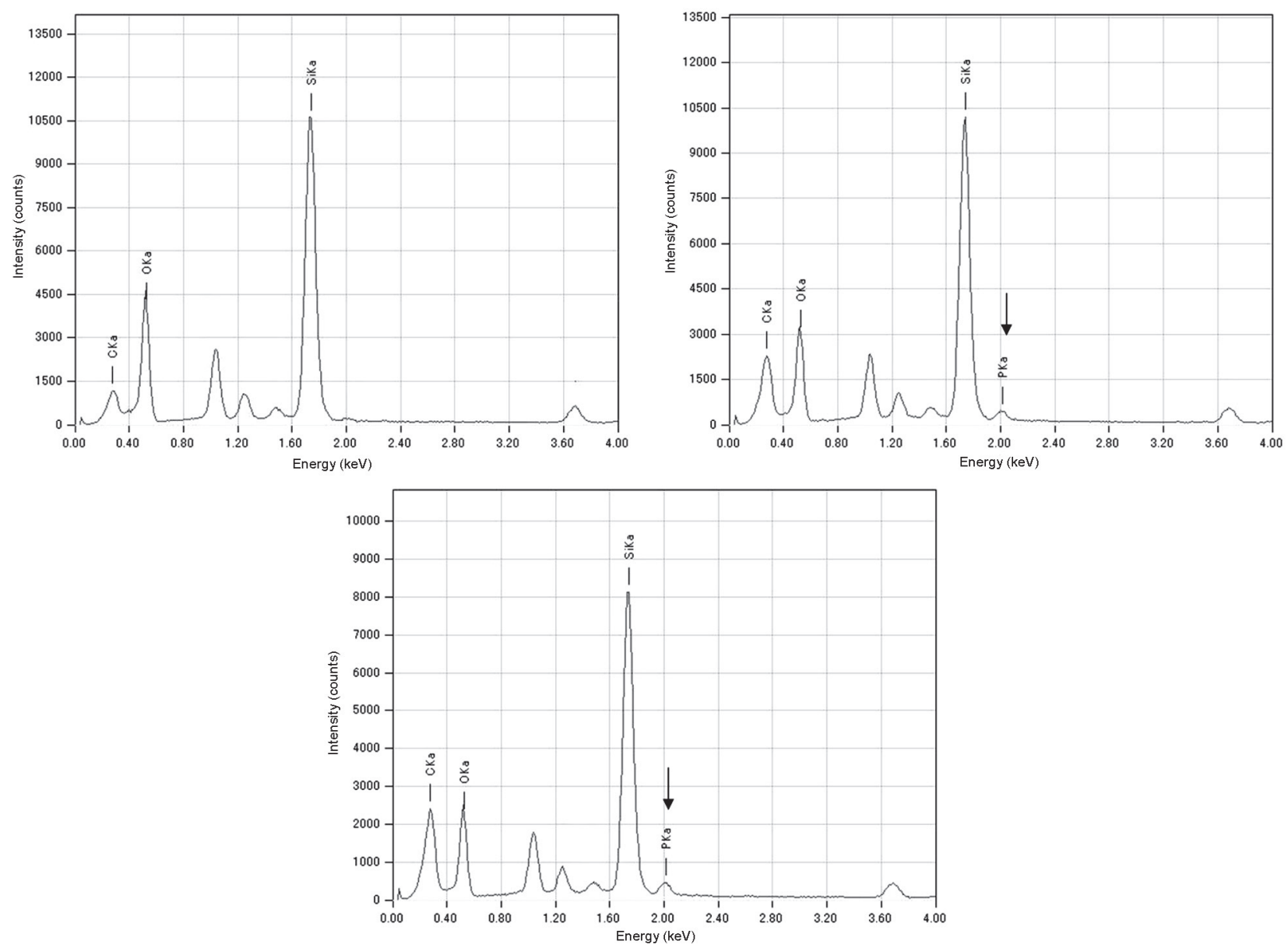

Fig. 3 EDX imaging of SE plate surfaces in control (upper left) and experimental groups before implantation (upper right), and that of experimental group after 12 weeks of implantation (lower). $\uparrow$ : Peaks for phosphate. 


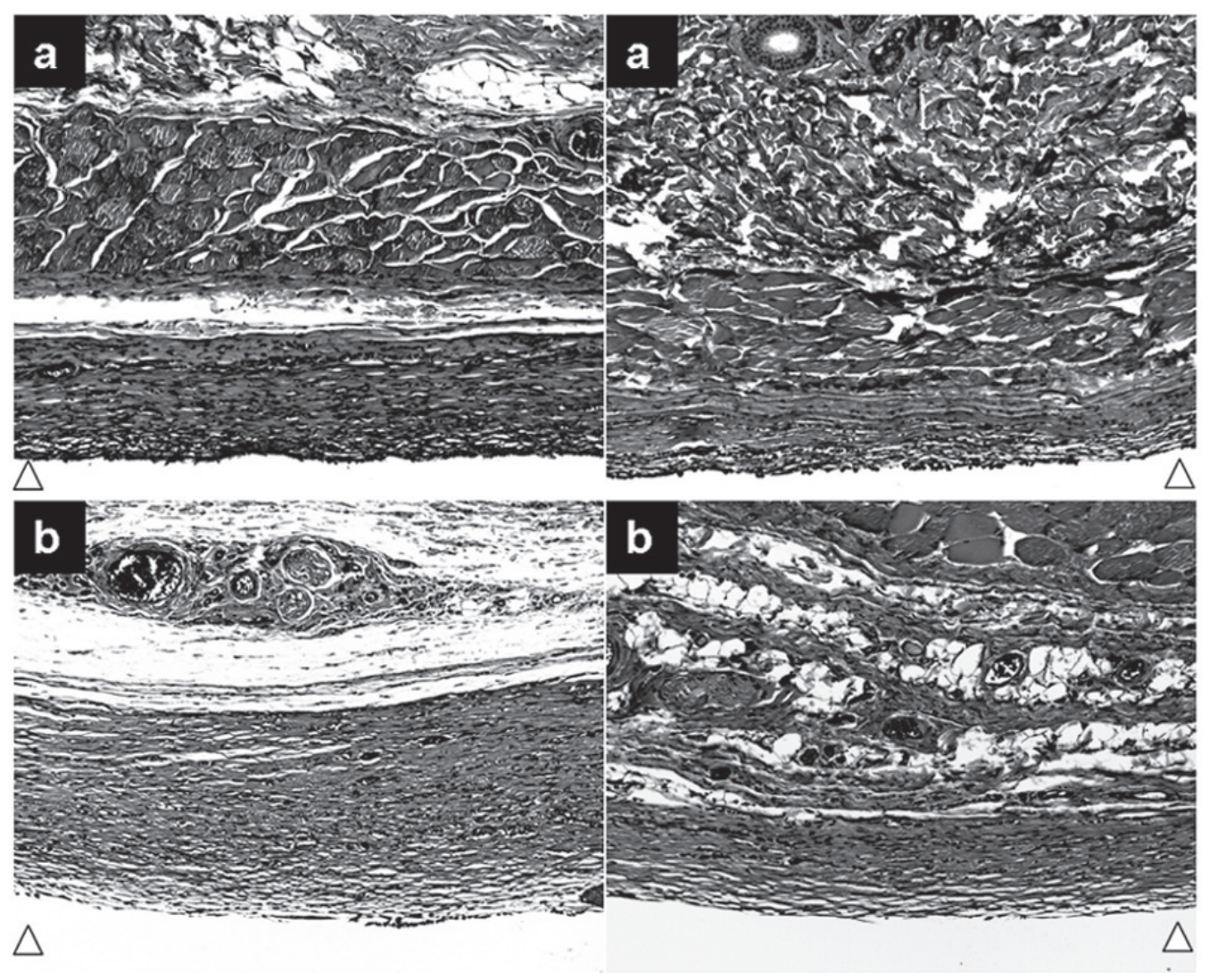

Fig. 4 Histological findings of surrounding fibrous connective tissues by H\&E staining $(\times 200)$ at 2 weeks (a) and 8 weeks (b). Left column: control group; right column: experimental group; $\Delta$ : SE plate surface.

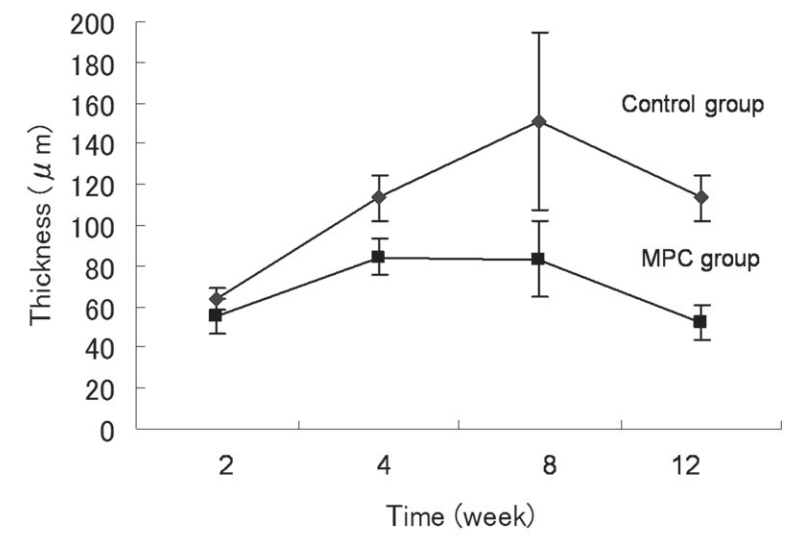

Fig. 5 Thicknesses of fibrous capsules at 2, 4, 8, and 12 weeks. Upper line graph: control group; lower line graph: experimental group.

In the experimental group, the thickness of fibrous tissue showed a gradual decrease at 8 and 12 weeks (Fig. 4). Moreover, at 4, 8, and 12 weeks, the surrounding fibrous tissue in the experimental group was thinner than that in the control group $(n=6$, $p<0.05$; Fig. 5). At 12 weeks, the fibrous tissue contained elastic fibers without inflammatory cell infiltration and capillary formation.

\section{Collagen staining}

Through a polarizing microscope, Sirius red and Fast green staining showed the collagen components of the fibrous tissue (Fig. 6). Figure 7 further shows the amounts of collagen fibers in fibrous capsules around the $\mathrm{SE}$ plates at 2, 4, 8, and 12 weeks for both experimental and control groups.

At 2 weeks, fibrous connective tissue was composed of numerous collagen fibers and scattered noncollagenous proteins such as proteoglycans and elastin in the experimental group. At 4 weeks, non-collagenous protein fibers gradually increased in both groups. Nonetheless, the amount of collagen in the experimental group was lower than that in the control group at 2 and 4 weeks $(n=6, p<0.01)$. At 8 weeks, fibrous tissue in the experimental group comprised a large number of elastic fibers and other non-collagenous components -in sharp contrast to the control group. At 12 weeks, there was a gradual increase in noncollagenous proteins in fibrous tissue for both groups, with the experimental group showing a higher level $(n=6, p<0.01)$. 

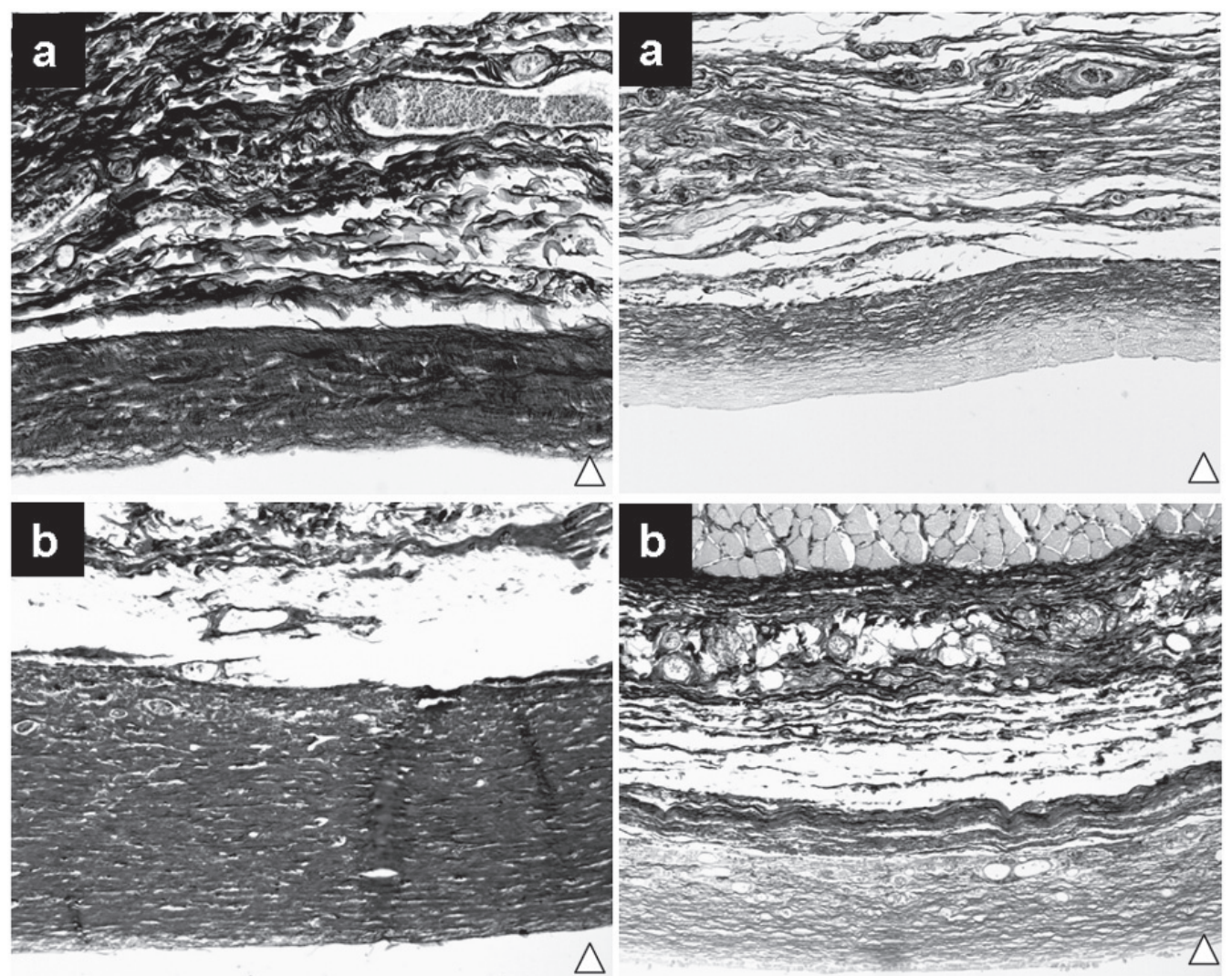

Fig. 6 Collagen staining of surrounding tissues with Sirius red and Fast green staining $(\times 200)$ at 2 weeks (a) and 8 weeks (b). Left column: control group; right column: experimental group; $\Delta$ : SE plate surface.

Analysis of cytoskeleton and cell adhesion

Figure 8 shows the extent of cell growth in both the experimental and control groups. In the control group, numerous KMST-6 cells with a spindle-shaped morphology - which is a typical fibroblastic shapewere observed, as well as signs of colony formation. In the experimental group, there were scattered roundshaped cell ${ }^{25)}$ over the surface but no signs of colony formation. Figure 9 shows the cell growth curves measured with MTT assay. It could be seen that cell proliferation on the MPC plate was slower than on the control plate.

In Fig. 10, phalloidin-positive cytoskeleton could be clearly seen. For the control group, KMST- 6 cells had a fully stretched shape with close contact to the SE plate. In the experimental group, round-shaped cells were seen on the coated surface with a large number of filopodia. The cells contained large amounts of short and dense skeletal structure.

Confocal images of immunohistochemical staining showed that vinculin expression was significantly weaker in the experimental group than in the control group (Fig. 11).

mRNA expression levels of fibronectin-1 and FAK The mRNA expressions of fibronectin-1 and FAK were

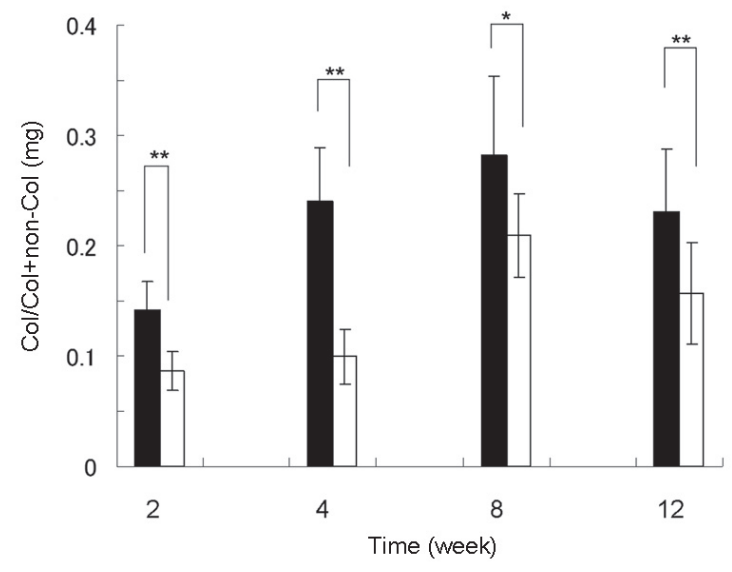

Fig. 7 Amounts of collagen fibers in fibrous capsules for experimental group (white vertical bars) and control group (black vertical bars). *: $p<0.05$; $* *: p<0.01$.

quantified using real-time PCR. Figure 12 shows the relative expression levels of fibronectin-1 and FAK mRNAs in KMST-6 cells that adhered to the experimental and control surfaces. The relative expression levels of fibronectin-1 and FAK mRNAs in 


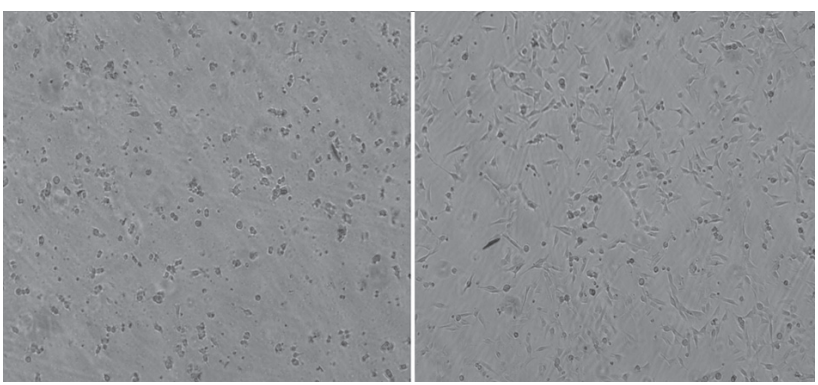

Fig. 8 Cell growth on SE plates coated and uncoated with MPC polymer in experimental group (left) and control group (right) respectively. Magnification at $\times 40$.

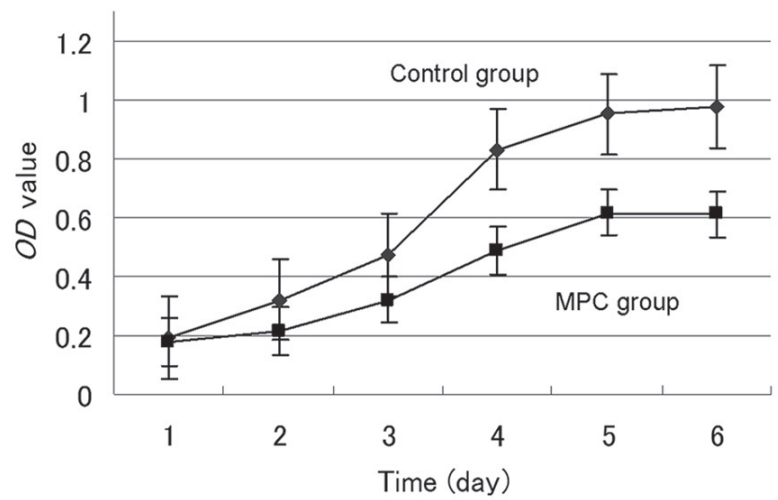

Fig. 9 Growth curves of cells on SE plates coated and uncoated with MPC polymer in experimental group (lower growth curve) and control group (upper growth curve) respectively.

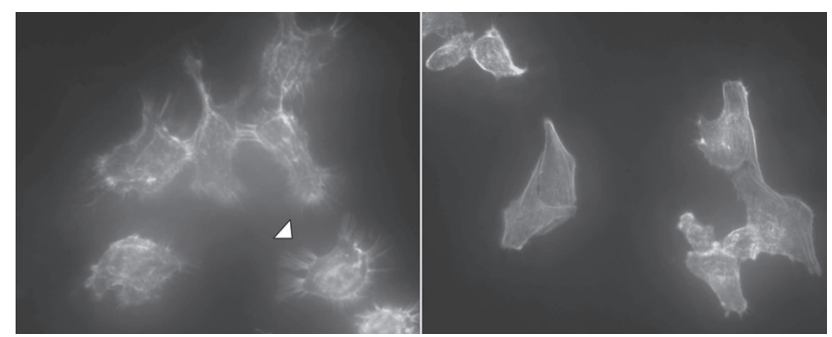

Fig. 10 KMST-6 cells stained with Alexa Fluor ${ }^{\circledR} 488$ conjugated to phalloidin. Left: experimental group, $\times 400$; right: control group, $\times 400 ; \Delta$ : filopodia.

the experimental group were clearly lower than those in the control group $(n=3, p<0.01)$.

Protein expression levels of ROCK1, cofilin, paxillin, and talin

Protein lysates of KMST-6 cells were analyzed using the Western blotting technique, and the results thereof are shown in Fig. 13. In the experimental group, the

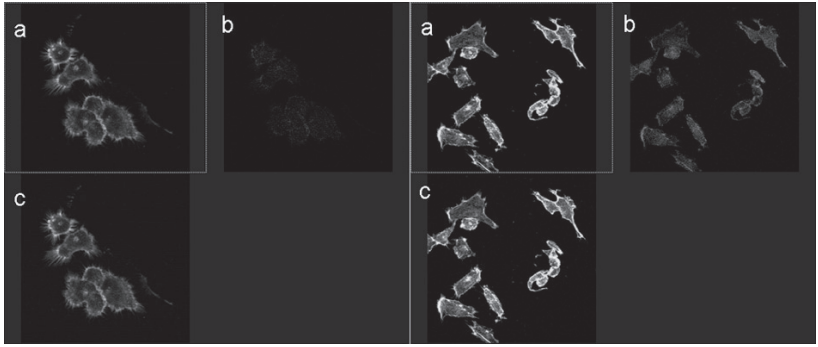

Fig. 11 Confocal images of vinculin expression by immunohistochemical staining in the experimental group (left, $\times 200$ ) and control group (right, $\times 200$ ). a: F-actin staining by phalloidin; b: vinculin expression; c: merged.

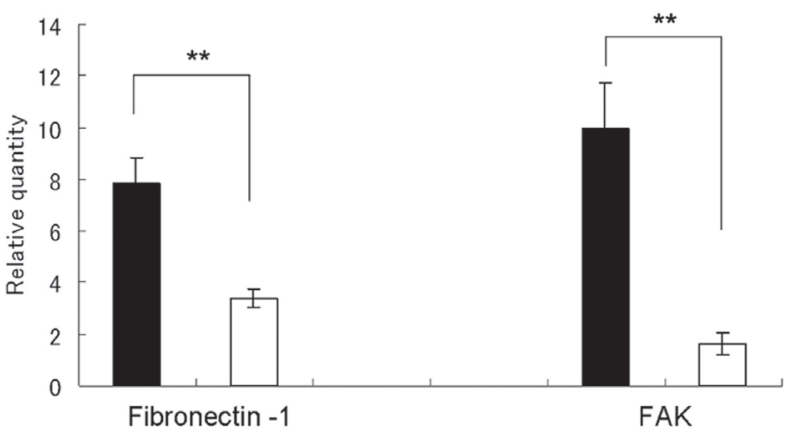

Fig. 12 Quantification of mRNA expression levels of fibronectin-1 and FAK by real-time PCR in experimental group (white vertical bars) and control group (black vertical bars). ${ }^{* *}: p<0.01$.

protein expressions of ROCK1, paxillin, and talin were significantly downregulated $(n=3, p<0.01)$. However, the expression of cofilin protein in the experimental group was significantly upregulated $(n=3, p<0.01)$.

\section{DISCUSSION}

Fibrous tissue formation after biomaterial implantation can lead to treatment failures, serious clinical problems, or loss of implanted device function ${ }^{26)}$. To prevent fibrous tissue formation, intervention methods include administration of 5-fluorouracil, interferonalpha 2b, and corticosteroids, UV irradiation or cryodesiccation therapy, and surgical treatment ${ }^{27)}$. However, these treatments are accompanied with various side effects, hence providing a strong impetus to improve the biocompatibility of implant materials to prevent unwanted fibrous tissue formation. Against this background, MPC polymer was developed as a material to inhibit harmful reactions at the interface between tissue and material ${ }^{6}$. Thus far, MPC polymer has attracted considerable attention because of its ability to influence cell reactions ${ }^{8}$. 
(a)
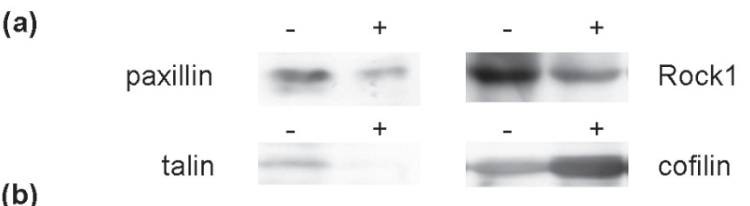

(b)

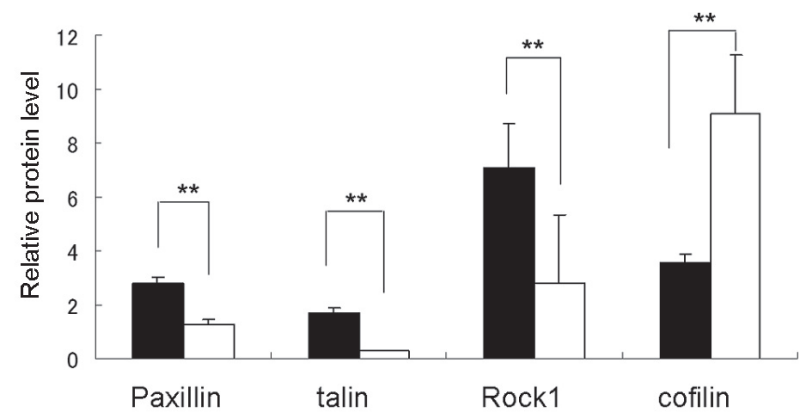

Fig. 13 Effects of MPC polymer on expressions of paxillin, talin, ROCK1, and cofilin proteins.

a: Western blot analysis shows protein levels of paxillin, talin, ROCK1, and cofilin in KMST-6 cells cultured on SE plates coated and uncoated with MPC polymer, where (-): control group and $(+)$ : experimental group.

b: Relative protein levels of paxillin, talin, ROCK1, and cofilin in experimental group (white vertical bars) and control group (black vertical bars). ${ }^{* *}: p<0.01$.

Inflammation and immune reactions around an implanted material will lead to development of fibrous tissue, and even increased fibrous tissue growth. Therefore, the thickness of fibrous tissue capsules and the collagen-synthetic response of fibroblasts are important parameters in the evaluation of a material's biocompatibility ${ }^{27)}$. In the present study, MPC polymercoated materials exhibited inhibition of fibrous tissue formation and collagen synthesis. In the same vein, the fibrous tissue surrounding MPC polymer-coated materials showed higher synthesis rates of noncollagenous proteins, such as elastin and proteoglycans, without an active inflammatory response. Our results thus showed that MPC polymer might have the capacity to regulate the quantity and quality of fibrous tissue formation. This meant that MPC polymer could contribute to preventing the deformation of and assisting in the smooth movement of implantation areas.

In general, biomaterials such as metals and bone graft materials emphasize cell adhesion. On the contrary, improvements to MPC have rendered it to be a biomaterial that mimics a biological cell membrane and which inhibits protein adsorption and cell adhesion $^{8,28)}$. The inhibition of cell adhesion has a negative impact on fibrous tissue formation ${ }^{29}$. Therefore, in this study, cultured cells on MPC polymer exhibited a round-shaped morphology and weaker expression of vinculin protein. Vinculin, acting through binding to F-actin, plays an important role in focal cell adhesion, in a manner similar to integrins ${ }^{30}$. At the same time, large amounts of filopodia immunofluorescently labeled with F-actin were observed in cultured cells on MPC polymer. These findings thus demonstrated the enhanced cell migration and weakened cell adhesion of the cultured cells on MPC polymer.

Fibronectin (FN) secreted from cells plays a major role in cell adhesion. In an in vitro study using bovine serum by Sawada et $a l .{ }^{31}$, MPC polymer surface registered a decrease in FN deposition. Similarly, results of the present study showed a significant reduction in $\mathrm{FN}$ expression of fibroblasts in contact with the MPC polymer. This meant that MPC polymer might have the capacity to diminish FN secretion upon contact with cells, thereby resulting in inhibited cell adhesion.

Integrin binding to $\mathrm{FN}$ leads to CAP formation, and at the same time induces downstream signaling. In other words, CAP formation mediated by $\mathrm{FN}$ induces signal transduction. Recent research has demonstrated a close relationship between integrin, vinculin, talin, and paxillin molecules and cell adhesion ${ }^{32}$. These molecules combine with fibronectin in the local microenvironment to form CAP. After CAP formation, cells perceive mechanical changes, and in response regulate their growth, secretion, plasticity and other physiological functions ${ }^{33}$.

In the present study, the expression of the downstream signaling molecules of $\mathrm{FN}$ was investigated using the Western blotting technique and a decrease in talin expression was detected. Owing to inhibited FN expression, there was a decrease in CAP formation and hence a decrease in talin expression - because inhibited FN expression affected downstream signal transduction. Besides, a downregulated paxillin expression reflected a cell migration trend, and this finding was augmented by a weaker expression of focal adhesion kinase (FAK). FAK represents a $125-\mathrm{kDa}$ protein which is localized to focal adhesion and which is activated by tyrosine phosphorylation in response to integrin clustering ${ }^{34)}$. Taken together, these results showed that the MPC polymer inhibited CAP formation by affecting downstream molecular signaling.

Enhanced cell migration on MPC polymer has been reported ${ }^{35)}$, whereby actin expression is an important parameter of cell migration capacity. ROCK1 and cofilin regulate actin metabolism through the Rho signaling pathway. ROCK1 maintains cell morphology by increasing the stability of actin-based stress fibers. ROCK1 also phosphorylates and activates LIM kinase, which phosphorylates cofilin. Cofilin stimulates actin depolymerization and changes in cell structure, and the phosphorylation of cofilin by LIM kinase suppresses its activity $^{36)}$. In this study, cultured cells on MPC polymer showed decreased ROCK1 and increased cofilin activity, leading to accelerated depolymerization of actin. Active actin metabolism with a large amount of filopodia formation represented enhanced cell 
migration, affecting collagen metabolism by fibroblasts as a result. Cell adhesion has a close relationship with collagen $^{37)}$. Okajima et al. found that with reduced secretion of Type I collagen - which is a major matrix component, fibrous tissue formation was prevented ${ }^{38}$.

Results of the present study suggested that fibrous tissue formation was prevented on MPC polymer-coated material because of decreased adsorption of proteins on the MPC polymer-coated silicon surface. In a study by Abraham et al. which investigated the adsorption of laminin, collagen, and fibronectin on polymer membrane surfaces, it was shown that the adsorption of proteins on biomaterial surfaces modulated cellular viability and restricted proliferation ${ }^{39)}$. In the context of the present study, the modulated cellular interaction with the MPC polymer-coated surface resulted in decreased secretion by and amplification of fibroblasts. Together with the concomitant regulation of signaling pathways such as Rho and FAK, scar formation was evidently attenuated.

In conclusion, this study showed the usefulness of artificial devices coated with MPC polymer, which mimicked a natural cell membrane. Nonetheless, for MPC polymer-coated implant materials to be used in a wide range of clinical applications, further research must be done to understand the role of direct metabolism exchange between cell membrane lipids and artificial phospholipids.

\section{ACKNOWLEDGMENTS}

This work is supported in part by the special coordination funds for Promoting Science and Technology from the Ministry of Education, Culture, Sports, Science, and Technology, Japan.

\section{REFERENCES}

1) Cohen ED. Prosthetic interfaces with the visual system: biological issues. J Neural Eng 2007; 4: R14-31.

2) Cornelis MA, Vandergugten S, Mahy P, De Clerck HJ, Lengelé B, D'Hoore W, Nyssen-Behets C. Orthodontic loading of titanium miniplates in dogs: microradiographic and histological evaluation. Clin Oral Implants Res 2008; 19: 1054-1062.

3) Brown MH, Shenker R, Silver SA. Cohesive silicone gel breast implants in aesthetic and reconstructive breast surgery. Plast Reconstr Surg 2005; 116: 768-779.

4) Waide V, Cristofolini L, Stolk J, Verdonschot N, Toni A. Experimental investigation of bone remodeling using composite femurs. Clin Biomech 2003; 18: 523-536.

5) Owen TA, Aronow M, Shalhoub V, Barone LM, Wilming L, Tassinari MS, Kennedy MB, Pockwinse S, Lian JB, Stein GS. Progressive development of the rat osteoblast phenotype in vitro: reciprocal relationships in expression of genes associated with osteoblast proliferation and differentiation during formation of the bone extracellular matrix. J Cell Physiol 1990; 143: 420-430.

6) Feng W, Zhu S, Ishihara K, Brash JL. Adsorption of fibrinogen and lysozyme on silicon grafted with poly (2methacryloyloxyethyl phosphorylcholine) via surfaceinitiated atom transfer radical polymerization. Langmuir 2005; 21: 5980-5987.
7) Ishihara K, Aragaki R, Ueda T, Watenabe A, Nakabayashi N. Reduced thrombogenicity of polymers having phospholipid polar groups. J Biomed Mater Res 1990; 24: 1069-1077.

8) Ishihara K, Nakabayashi N, Fukumoto K, Aoki J. Improvement of blood compatibility on cellulose dialysis membrane. I. Grafting of 2-methacryloyloxyethyl phosphorylcholine on to a cellulose membrane surface. Biomaterials 1992; 13: 145-149.

9) Xu Y, Takai M, Ishihara K. Suppression of protein adsorption on a charged phospholipid polymer interface. Biomacromolecules 2009; 10: 267-274.

10) Seo JH, Matsuno R, Konno T, Takai M, Ishihara K. Surface tethering of phosphorylcholine groups onto poly (dimethylsiloxane) through swelling-deswelling methods with phospholipids moiety containing ABA-type block copolymers. Biomaterials 2008; 29: 1367-1376.

11) Kyomoto M, Moro T, Saiga K, Miyaji F, Kawaguchi $H$, Takatori Y, Nakamura K, Ishihara K. Lubricity and stability of poly(2-methacryloyloxyethyl phosphorylcholine) polymer layer on Co-Cr-Mo surface for hemi-arthroplasty to prevent degeneration of articular cartilage. Biomaterials 2010; 31: 658-668.

12) Huang XD, Yao K, Zhang H, Huang XJ, Xu ZK. Surface modification of silicone intraocular lens by 2methacryloyloxyethyl phosphoryl-choline binding to reduce Staphylococcus epidermidis adherence. Clin Experiment Ophthalmol 2007; 35: 462-467.

13) Mang A, Pill J, Gretz N, Kränzlin B, Buck H, Schoemaker M, Petrich W. Biocompatibility of an electrochemical sensor for continuous glucose monitoring in subcutaneous tissue. Diabetes Technol Ther 2005; 7: 163-173.

14) Ho SP, Nakabayashi N, Iwasaki $Y$, Boland T, LaBerge M. Frictional properties of poly(MPC-co-BMA) phospholipid polymer for catheter applications. Biomaterials 2003; 24: 5121-5129.

15) Konno T, Kawazoe N, Chen G, Ito Y. Culture of mouse embryonic stem cells on photoimmobilized polymers. J Biosci Bioeng 2006; 102: 304-310.

16) Hinz B, Gabbiani G. Mechanisms of force generation and transmission by myofibroblasts. Curr Opin Biotechnol 2003; 14: 538-546.

17) Bell WT, Chalian VA, Moore BK. Polydimethyl siloxane materials in maxillofacial prosthetics: evaluation and comparison of physical properties. J Prosthet Dent 1985; 54: 404-410.

18) Lewis DH, Castleberry DJ. An assessment of recent advances in external maxillofacial materials. J Prosthet Dent 1980; 43: 426-432.

19) Chang PP, Hansen NA, Phoenix RD, Schneid TR. The effects of primers and surface bonding characteristics on the adhesion of polyurethane to two commonly used silicone elastomers. J Prosthodont 2009; 18: 23-31.

20) Kyomoto M, Moro T, Miyaji F, Konno T, Hashimoto M, Kawaguchi H, Takatori Y, Nakamura K, Ishihara K. Enhanced wear resistance of orthopaedic bearing due to the cross-linking of poly(MPC) graft chains induced by gammaray irradiation. J Biomed Mater Res B 2008; 84: 320-327.

21) Sibarani J, Takai M, Ishihara K. Surface modification on microfluidic devices with 2-methacryloyloxyethyl phosphorylcholine polymers for reducing unfavorable protein adsorption. Colloids Surf B Biointerfaces 2007; 54: 88-93.

22) Lehle K, Lohn S, Reinerth GG, Schubert T, Preuner JG, Birnbaum DE. Cytological evaluation of the tissue-implant reaction associated with subcutaneous implantation of polymers coated with titaniumcarboxonitride in vivo. Biomaterials 2004; 25: 5457-5466.

23) Wang WD, Xu XM, Chen Y, Jiang P, Dong CZ, Wang Q. 
Apoptosis of human Burkitt's lymphoma cells induced by 2$\mathrm{N}, \mathrm{N}$-diethylaminocarbonyloxymethyl-1-diphenylmethyl-4(3,4,5-trimethoxybenzoyl) piperazine hydrochloride (PMS1077). Arch Pharm Res 2009; 32: 1727-1736.

24) Heckmann L, Schlenker HJ, Fiedler J, Brenner R, Dauner M, Bergenthal G, Mattes T, Claes L, Ignatius A. Human mesenchymal progenitor cell responses to a novel textured poly(L-lactide) scaffold for ligament tissue engineering. J Biomed Mater Res B 2007; 81: 82-90.

25) Nam K, Kimura T, Kishida A. Physical and biological properties of collagen-phospholipid polymer hybrid gels. Biomaterials 2007; 28: 3153-3162.

26) Mandalia V, Eyres K, Schranz P, Toms AD. Evaluation of patients with a painful total knee replacement. J Bone Joint Surg Br 2008; 90: 265-271.

27) Al-Attar A, Mess S, Thomassen JM, Kauffman CL, Davison SP. Keloid pathogenesis and treatment. Plast Reconstr Surg 2006; 117: 286-300.

28) Watanabe J, Eriguchi T, Ishihara K. Cell adhesion and morphology in porous scaffold based on enantiomeric poly(lactic acid) graft-type phospholipid polymers. Biomacromolecules 2002; 3: 1375-1383.

29) Shigeta M, Tanaka T, Koike N, Yamakawa N, Usui M. Suppression of fibroblast and bacterial adhesion by MPC coating on acrylic intraocular lenses. J Cataract Refract Surg 2006; 32: 859-866.

30) Mierke CT. The role of vinculin in the regulation of the mechanical properties of cells. Cell Biochem Biophys 2009; 53: $115-126$.

31) Sawada S, Sakaki S, Iwasaki Y, Nakabayashi N, Ishihara K. Suppression of the inflammatory response from adherent cells on phospholipid polymers. J Biomed Mater Res A
2003; 64: 411-416.

32) Machesky L, Jurdic P, Hinz B. Grab, stick, pull and digest: the functional diversity of actin-associated matrix-adhesion structures. Workshop on Invadopodia, Podosomes and Focal Adhesions in Tissue Invasion. EMBO Rep 2008; 9: 139-143.

33) Ballestrem C, Hinz B, Imhof BA, Wehrle-Haller B Marching at the front and dragging behind: differential alphaVbeta3-integrin turnover regulates focal adhesion behavior. J Cell Biol 2001; 155: 1319-1332.

34) Tomar A, Schlaepfer DD. Focal adhesion kinase: switching between GAPs and GEFs in the regulation of cell motility. Curr Opin Cell Biol 2009; 21: 676-683.

35) Kaji H, Kawashima T, Nishizawa M. Patterning cellular motility using an electrochemical technique and a geometrically confined environment. Langmuir 2006; 22: 10784-10787.

36) Dubash AD, Menold MM, Samson T, Boulter E, GarcíaMata R, Doughman R, Burridge K. Chapter 1. Focal adhesions: new angles on an old structure. Int Rev Cell Mol Biol 2009; 277: 1-65.

37) Taubenberger AV, Woodruff MA, Bai H, Muller DJ, Hutmacher DW. The effect of unlocking RGD-motifs in collagen I on pre-osteoblast adhesion and differentiation. Biomaterials 2010; 31: 2827-3285.

38) Okajima Y, Saika S, Sawa M. Effect of surface coating an acrylic intraocular lens with poly(2-methacryloyloxyethyl phosphorylcholine) polymer on lens epithelial cell line behavior. J Cataract Refract Surg 2006; 32: 666-671.

39) Abraham S, Brahim S, Guiseppi-Elie A. Molecularly engineered hydrogels for implant biocompatibility. Conf Proc IEEE Eng Med Biol Soc 2004; 7: 5036-5039. 